\title{
A different ultrastructural face of ribbon synapses in the rat retina
}

\author{
Artur Pałasz ${ }^{1}$, Łukasz Mielańczyk ${ }^{2^{*}}$, Natalia Matysiak ${ }^{2^{*}}$, Yolanda Segovia ${ }^{3}$ \\ Mariia Savchyna ${ }^{4}$, Kinga Mordecka-Chamera ${ }^{1}$, John J. Worthington ${ }^{5}$
}

\footnotetext{
${ }^{1}$ Department of Histology, School of Medicine in Katowice, Medical University of Silesia, ul. Medyków 18, 40-752, Katowice, Poland

${ }^{2}$ Department of Histology and Cellular Pathology, School of Medicine with Division of Dentistry in Zabrze, Medical University of Silesia, ul. Jordana 19, 41-808, Zabrze, Poland

${ }^{3}$ Department of Biotechnology, University of Alicante, Apartado 99, 03080, Alicante, Spain

${ }^{4}$ Department of Histology and Embryology, Bogomolets National Medical University, Kyiv, Ukraine

${ }^{5}$ Division of Biomedical and Life Sciences, Faculty of Health and Medicine, Lancaster University, Lancaster, LA1 4YQ, UK

*both authors contributed equally to this work
}

Abstract

Ribbon synapses located exclusively within retinal, cochlear and vestibular connections belong to the most interesting cellular structures but their molecular nature and functions had remain unclear. The study has provided a descriptive morphological analysis of rat eye ribbon synapses using high resolution transmission electron microscopy (TEM). An original collection of untypical, rarely present in the literature sagittal or tangential sections through the single RIBEYE domain of the particular ribbon have been delivered.

Key words: ribbon synapse, RIBEYE, retina, eye 


\section{Introduction}

Ribbon synapses are peculiar and intriguing intercellular sensory connections located exclusively within retina photoreceptors, vestibular sensory cells, pinealocytes and the inner hair cells of the organ of Corti (Laver and Matsubara 2017, Satz and Campbell 2008, Spiwoks-Becker et al. 2008). Despite being first described as early as the 1950s by Sjostrand and Smith, the neurochemistry and functions of synaptic ribbons had remain delusive. However, recent studies have now shown these cells contribute to replenishing release sites, ensure high complement and spatial coupling of vesicular release sites and CaV 1.4 calcium channels at the active zone, while helping multivesicular release and synaptic vesicle endocytosis from cell membranes (Maxeiner et al. 2016, Mehta et al. 2013). Recent hypotheses suggest that the ribbon forms a reservoir of primed glutamate vesicles in very close contact with the presynaptic membrane. Synapses in both the retina and inner ear have to produce impulses at very high rates, releasing as many as 500 vesicles per second (Schmitz 2009). The pool of vesicles tethered to the ribbon facilitates this fast signal transduction in sensory cells (Pelassa et al. 2014). At retinal ribbon synapses, the rich calcium channel invagination of the presynaptic membrane is a site of glutamate exocytosis (Innocenti and Heidelberger 2008), while the active zone cytomatrix proteins Bassoon, Piccolo, and the dystroglycan ligand - pikachurin are considered to play a crucial role in the formation and activity of the whole structure (Dick et al. 2003, Sato et al. 2008). The released neurotransmitter binds to molecules of the metabotropic glutamate receptor 6 (mGluR6) on the ON-bipolar cells. In turn 
both horizontal cells and OFF-bipolar cells have ionotopic glutamate receptors (Hartveit et al. 2018) associated with the postsynaptic proteinsProSAP1/Shank2 and Homer1 (Brandstätter et al. 2004). Synaptic ribbons are very dynamic cellular structures, able to grow and shrink with circadian changes in functional demands such as during the daytime or under increased illumination (Regus-Leidig and Brandstätter 2012,Oesch and Diamond 2011, Hull et al. 2006). Despite the ongoing histological studies on retinal synaptology, there is still an insufficient number of morphological reports depicting the ultrastructure of ribbon synapses. A certain knowledge gap exists concerning satisfactory high yield EM visualization of the $\operatorname{RIBEYE}(A) /(B)$ protein complexes that form the ribbons. In the current study, we aim to provide a descriptive structural analysis of rat eye ribbon synapses using high resolution transmission electron microscopy (TEM).

\section{Methods}

Study was carried out on adult (5 months old) male Sprague-Dawley rats $(n=2)$. All experimental procedures were approved by the Local Bioethical Committee and were conducted in a manner consistent with $\mathrm{NIH}$ Guidelines for Care and Use of Laboratory Animals. Animals were anesthetized using 2\% isoflurane and oxygen flow under spontaneous breathing, were sacrificed and four eyeballs enucleated, opened and the vitreous body removed. The eye specimens were fixed in $2.5 \%$ glutaraldehyde in cacodylate buffer $(\mathrm{pH} 7.4)$ for $4 \mathrm{hrs}$ at room temperature followed by washing in the same buffer several times. Subsequently, samples were dehydrated in a graded series of ethanol, cleared in propylene oxide and embedded in Epon 812, and polymerized for $48 \mathrm{hrs}$ at $60^{\circ} \mathrm{C}$. Ultrathin sections were cut with a diamond knife (450; RMC, Tucson, USA) using a Reichert OmU-3 ultramicrotome, mounted on 300-mesh copper grids, and stained with uranyl acetate and lead citrate 
using a Leica EM AC 20 stainer. Ultrathin sections of retina were evaluated in a Tecnai G2 12 Spirit BioTWIN Transmission Electron Microscope (FEI, Eindhoven, The Netherlands) at $120 \mathrm{kV}$. Images from representative retinal regions were captured with a Morada CCD camera (Olympus Soft Imaging System Solutions GMBH, Münster, Germany). The length and thickness of the cross sectioned ribbons taken from 50 cells were measured and analyzed. The quantification derived just from ribbons were the arciform density and internal striations are visible.

\section{Results and discussion}

We found considerable differences both in the ribbon size and in the number of adjacent vesicle pools within the examined synaptic connections of the rat outer plexiform layer (Fig 1.). This confirms the presence of a previously reported distinct structural diversity of ribbons in animal photoreceptors (Regus-Leidig and Brandstätter 2012). The thickness of photoreceptor synaptic ribbons was relatively constant, we found no correlation between ribbon length and its thickness (Fig 1.) The evidence of various stereological forms of the ribbons (Fig 1.) including spherical figures (Fig.2.B) may reflect the dynamic process of their assembly/disassembly in the receptor cells (Mercer and Thoreson 2011). More importantly our study also depicts the subtle ultrastructure of the cross sectioned ribbon scaffold (Fig.2, A,C,D) with a resolution high enough to show the previously described layers of RIBEYE(A)/(B) domains (Magupalli et al. 2008). RIBEYE protein is an intrinsically dynamic and fundamental component of the synaptic ribbon - its deletion makes synapses ribbonless (Chen et al. 2018, Mehta et al. 2013). Its molecule consists of two domains: an $\mathrm{N}$-terminal proline-rich A-domain that appears to have a predominantly structural role, and a C-terminal B-domain, that points to the cytoplasmic face of the synaptic ribbon where it binds NADH (Sheets et al. 2014, 
Magupalii et al. 2008, Schmitz et al. 2000) or interacts with some proteins such as Munc 119 and GCAP2 (Vollrath and Spiwoks-Becker 1996). Interestingly, a B-subunit is analogous to C-terminal binding protein $2 s$ (CtBP2s)-a splice isoform of the transcriptional corepressor CtBP2 (Verger et al. 2006). The RIBEYE domains interplay between three binding sites in their A-domain and two binding sites in their B-section that may regulate the assembly and dynamics of synaptic ribbons (RegusLeidig et al. 2010). Number of other proteins different from RIBEYE have been described as components of synaptic ribbons and ribbon synapses (Tom Dieck and Brandstätter. 2006). In the present study we showed that inner leaflets of the ribbon are electron-lucent and outer ones are in turn electron dense. This intriguingly matching the current model for RIBEYE domain organization by Magupalli et al. 2008, therefore we speculate that RIBEYE-B domain corresponds to the electrondense part and the inward-facing electron-lucent part corresponds to RIBEYE-A domain. This is supported by CtBP2s crystal structure data and the notion that the Adomain is more flexible (Nardini et al. 2003. Schmitz et al. 2000). The structural orientation of scaffold proteins is slightly different than previously described in mice (Schmitz 2009, Sterling and Matthews 2005). Furthermore, our study has delivered a set of unique, sagittal or tangential sections through the single RIBEYE domain of the particular ribbon (Fig 2.). Irregular shaped, elongated areas with a lower or higher electron density represent the sections across $\operatorname{RIBEYE}(A)$ and $R I B E Y E(B)$ sheets respectively. These kinds of TEM images are rarely present in the literature, and hence we have decided to present these original electronograms to the scientific community. Nevertheless, we would like to point out some limitations of TEM in the study on ribbon molecular composition. To really address the question of RIBEYEorientation within the synaptic ribbon with electron microscopical methods including 
CryoEM, one needs to perform postembedding immunogold labeling with antibodies specific for the RIBEYE(A)- and (B)-domain and quantify their respective distance to the centre of the cross-sectioned synaptic ribbon. The results presented here can be appropriately used as a starting point to generate a more systematic analysis of retinal ribbons, for instance comparing their ultrastructure in photoreceptors, operating a distinction between rods and cones, as well as including those of the inner plexiform layer. Since the physiology of glutamate release from rod bipolar cells has been well studied, functional correlations of ultrastructural data definitely are possible and useful.

\section{REFERENCES}

Brandstätter J.H., Dick O. \& Boeckers T.M., 2004. The postsynaptic scaffold proteinsProSAP1/Shank2 and Homer1 are associated with glutamate receptor complexes at rat retinal synapses. J Comp Neurol,475(4),551-563.

Chen Z., Chou S.W. \& McDermott B.M. Jr., 2018. Ribeye protein is intrinsically dynamic but is stabilized in the context of the ribbon synapse. J. Physiol., 596 (3), 409-421.

Dick O., tom Dieck S., Altrock W.D., Ammermüller J., Weiler R., Garner C.C., Gundelfinger E.D. \&Brandstätter J.H., 2003. The presynaptic active zone protein bassoon is essential for photoreceptor ribbon synapse formation in the retina. Neuron, 37(5),775-786.

Hartveit E., Zandt B.J., Madsen E., Castilho Á., Mørkve S.H.\&Veruki M.L., 2018. AMPA receptors at ribbon synapses in the mammalian retina: kinetic models and molecular identity. Brain Struct. Funct., 223(2),769-804..

Hull C., Studholme K., Yazulla S.\& von Gersdorff H., 2006. Diurnal changes in exocytosis and the number of synaptic ribbons at active zones of an ON-type bipolar cell terminal. J. Neurophysiol.96, 2025-2033..

Innocenti B., Heidelberger R., 2008. Mechanisms contributing to tonic release at the cone photoreceptor ribbon synapse. J. Neurophysiol., 99(1), 25-36. 
Laver C.R.J., Matsubara J.A., 2017. Structural divergence of essential triad ribbon synapse proteins among placental mammals - Implications for preclinical trials in photoreceptor transplantation therapy. Exp. Eye Res., 159, 156-167.

Magupalli V.G.. Schwarz K., Alpadi K., Natarajan S., Seigel G.M. \&Schmitz F., 2008. Multiple RIBEYE-RIBEYE interactions create a dynamic scaffold for the formation of synaptic ribbons. J. Neurosci., 28(32),7954-7967.

Maxeiner S., Luo F., Tan A., Schmitz F., Südhof T.C., 2016. How to make a synaptic ribbon: RIBEYE deletion abolishes ribbons in retinal synapses and disrupts neurotransmitter release. EMBO J., 35(10),1098-1114.

Mercer A. J. \& Thoreson, W. B., 2011. The dynamic architecture of photoreceptor ribbon synapses: cytoskeletal, extracellular matrix, and intramembrane proteins. Vis. Neurosci., 28, 453-471.

Mehta B., Snellman J., Chen S., Li W., Zenisek D., 2013. Synaptic ribbons influence the size and frequency of miniature-like evoked postsynaptic currents. Neuron, 77(3), 516-527.

Nardini M, Valente C, Ricagno S, Luini A, Corda D, Bolognesi M. CtBP1/BARS Gly172-->Glu mutant structure: impairing NAD(H)-binding and dimerization. Biochem. Biophys. Res. Commun. , 27 (1):70-4.

Oesch N.W., Diamond J.S., 2011. Ribbon synapses compute temporal contrast and encodeluminance in retinal rod bipolar cells. Nat. Neurosci., 14(12), 1555-1561.

Pelassa I., Zhao C., Pasche M., Odermatt B., Lagnado L., 2014. Synaptic vesicles are"primed" for fast clathrin-mediated endocytosis at the ribbon synapse. Front. Mol. Neurosci., 7, 91.

Regus-Leidig H., Brandstätter, J. H., 2012. Structure and function of a complex sensory synapse. Acta Physiol., 204, 479-486.

Regus-Leidig H., Specht D., Tom Dieck S., Brandstatter J.H., 2010. Stability of active zone components at the photoreceptor ribbon complex. Mol. Vis., 16: 2690-2700.

Sato S., Omori Y., Katoh K., Kondo M., Kanagawa M., Miyata K., Funabiki K., Koyasu T., Kajimura N., Miyoshi T., Sawai H., Kobayashi K., Tani A., Toda T., Usukura J., Tano Y., Fujikado T., Furukawa T. (2008). Pikachurin, a dystroglycan 
ligand, is essential for photoreceptor ribbon synapse formation. Nat. Neurosci., 11(8),923-931.

Satz J.S. \&Campbell K.P., 2008. Unraveling the ribbon synapse. Nat. Neurosci., 11(8), 857-859.

Schmitz F., 2009. The making of synaptic ribbons: how they are built and what they do. Neuroscientist, 15(6),611-624.

Schmitz F., Konigstorfer A., Sudhof T.C., 2000. RIBEYE, a component of synaptic ribbons: a protein's journey through evolution provides insight into synaptic ribbon function. Neuron 28: 857-872.

Sheets L, Hagen MW, Nicolson T., 2014. Characterization of Ribeye subunits in zebrafish hair cells reveals that exogenous Ribeye B-domain and CtBP1 localize to the basal ends of synaptic ribbons. PLoS One, 10 (9):e107256.

Spiwoks-Becker I., Maus C., tom Dieck S., Fejtová A., Engel L., Wolloscheck T., Wolfrum U., Vollrath L., Spessert R., 2008. Active zone proteins are dynamically associated with synaptic ribbons in rat pinealocytes. Cell Tissue Res., 333(2),185195.

Sterling P., Matthews G., 2005. Structure and function of ribbon synapses. Trends Neurosci., 28(1),20-29.

Tom Dieck S., Brandstätter J.H., 2006. Ribbon synapses of the retina. Cell Tissue Res., 326(2), 339-346.

Verger A., Quinlan K.G., Crofts L.A., Spanò S., Corda D., Kable E.P., Braet F., Crossley M., 2006. Mechanisms directing the nuclear localization of the CtBP family proteins. Mol. Cell. Biol., 26(13):4882-94.

Vollrath L. \& Spiwoks-Becker I., 1996. Plasticity of retinal ribbon synapses. Microsc. Res. Tech., 35, 472-487. 
Figure captions

Fig. 1. Utrastructure of the retinal ribbon synapses and their basic 2-D qualitative analysis. Classical frontal view of the synaptic ribbons. The cytoplasm of the photoreceptor cell with the ribbons surrounded by synaptic vesicles (A-C, blue). Sections through the processes of both horizontal (yellow) and bipolar (red) cells are present (D-F). Ribbons are located close to the arciform density in the presynaptic membrane. Scale bars: $500 \mathrm{~nm}(A, F) ; 200 \mathrm{~nm}(B, C, D, E)$. Mean thickness of the ribbons (bars, left scale) in comparizon to their length (curve, right scale). Each bar and appropriate peak represent a single ribbon that have met the criteria mentioned in the methods. Double $y$-axis scales were provided to increase the graph clarity. The measurements reveal no correlation between ribbon length and thickness in the rat retina (mean values \pm SEM).

Fig. 2. Sections of the ribbons at diverse stereological planes. The sagittally sectioned RIBEYE protein domains are visible $(A, C, D)$. The area with a lower electron density (a.) represents inner $\operatorname{RIBEYE}(A)$ layer, whereas higher density (b.) is characteristic for the outer RIBEYE(B) sheet. Cross section of the spherical ribbon (B, asterisk). Scale bars; $1 \mu \mathrm{m}(A), 500 \mathrm{~nm}(B, C, D), 100 \mathrm{~nm}$ (inset). Schematic 3Dmodelling of the ribbon sections $(E)$, sagittal or partly tangential sections through the domains: RIBEYE(A) from the D5 (a.) and RIBEYE(B) from D6 (b.). The RIBEYE domains $A$ and $B$ are marked in red and blue, respectively. They were also spread apart virtually just to visualize the most probable 3-D situation of the aforementioned areas $(a, b)$. An outline molecular structure of the ribbon with the spatial orientation of RIBEYE domains including site of RIBEYE(B)-GCAP2 regulatory interaction and NADH binding subdomain. 